\title{
SÍNDROME DO NINHO VAZIO: EXPERIÊNCIAS DE IDOSOS INTEGRANTES DA UNIVERSIDADE DA MATURIDADE DO AMAPÁ
}

\author{
EMPTY NEST SYNDROME: EXPERIENCES OF ELDERLY PEOPLE AT \\ THE UNIVERSITY OF MATURITY PROJECT IN AMAPÁ
}

\section{SÍNDROME DEL NIDO VACÍO: EXPERIENCIAS DE ADULTOS MAYORES DEL PROYECTO UNIVERSIDAD DE MADUREZ DE AMAPÁ}

\author{
Rafaela Ingrid Mota Dos Santos* \\ Anneli Mercedes Celis De Cárdenas** \\ Rosilda Alves Da Silva Isla Chamilco*** \\ Nely Dayse Santos Da Mata**** \\ Luzilena De Sousa Prudêncio***** \\ Maria Virgínia Filgueiras De Assis Mello******
}

\begin{abstract}
RESUMO
Objetivo: Identificar como idosos integrantes do Projeto Universidade da Maturidade do Amapá (UMAP) vivenciam o fenômeno da Síndrome do Ninho Vazio. Material e Método: Trata-se de estudo exploratório e descritivo, com abordagem qualitativa. A coleta de dados ocorreu com sete idosos, com idades entre 63 e 83 anos, por meio de entrevista semiestruturada, contemplando uma questão central: conte-me como foi a sua experiência mediante a saída dos filhos de casa? Para análise dos dados adotou-se a análise de conteúdo de Bardin, subsidiada pelo software ATLAS.ti versão 8.0 que por meio de uma matriz de análise originou três categorias temáticas. Resultados: As categorias resultaram de um processo analítico e explicativo da experiência vivenciada pelos idosos e compreenderam três momentos: A vida sendo invadida pela solidão, Beneficiandose da liberdade e Alçando novos voos. Conclusão: Em resposta ao objetivo do estudo, foi possível identificar que a experiência do ninho vazio vivenciada pelos idosos perpassou por diferentes fases, entretanto o convívio
\end{abstract}

*Enfermeira. Secretaria do Estado de Saúde do Amapá. Macapá - Amapá, Brasil, ORCID: https://orcid.org/0000-0002-02009346. Email: rafaelaingridmota@gmail.com Autor da correspondência.

**Enfermeira. Doutora em Enfermagem. Curso de Bacharelado em Enfermagem, Universidade Federal do Amapá, Macapá Amapá, Brasil, ORCID: https://orcid.org/0000-0002-6581-4326 Email: celis.unifap@gmail.com

***Enfermeira. Doutora em Enfermagem. Curso de Bacharelado em Enfermagem, Universidade Federal do Amapá, Macapá Amapá, Brasil. ORCID: https://orcid.org/0000-0003-2861-5907 Email: chamilco@yahoo.com.br

****Enfermeira. Doutora em Ciências, Curso de Bacharelado em Enfermagem, Universidade Federal do Amapá, Macapá Amapá, Brasil, ORCID: http://orcid.org/0000-0002-0245-8141 Email: nelymata@uol.com

*****Enfermeira. Doutora em Saúde Coletiva, Curso de Bacharelado em Enfermagem, Universidade Federal do Amapá, Macapá - Amapá, Brasil, ORCID: https://orcid.org/0000-0003-3364-07 Email: luzilenarohde@hotmail.com

******Enfermeira. Doutora em Ciências, Curso de Bacharelado em Enfermagem, Universidade Federal do Amapá, Macapá Amapá, Brasil, ORCID: https://orcid.org/0000-0002-5260-942X Email: virginia@unifap.br Autor da correspondência 
na UMAP viabilizou superar a solidão e transformar esta experiência em oportunidade de fortalecimento e crescimento.

Palavras-chave: Idoso; Relaçôes Familiares; Síndrome do Ninho Vazio; Universidades; Acolhimento.

\begin{abstract}
Objective: To identify how elderly members who participate of the Project University of Maturity (UMAP), of the Federal University of Amapá in Brazil, experience the Empty Nest Syndrome phenomenon. Materials and Methods: Qualitative, exploratory and descriptive study, using narratives to understand the story of the participants. Data collection took place with seven elderly people, aged between 63 and 83 years, through semistructured interviews, an focusing on a central question: How was your experience when your children grew up and left home? For data analysis, Bardin's content analysis was adopted, supported by the software ATLAS. ti version 8.0. To guarantee methodological rigor, the principles of credibility, reliability, confirmability and transferability were respected. Results: The resulting categories emerged from an analytical and explanatory process of the experience lived by the elderly and consisted of three moments: life invaded by loneliness, benefiting from freedom and taking new chances. Conclusion: The study shows that the empty nest experience undergoes different phases. However, the experience at UMAP made it possible to overcome loneliness and transform this experience into an opportunity for strengthening and growth.
\end{abstract}

Key words: Elderly People; Family Relationships; Empty Nest Syndrome; Universities; User Embracement.

\title{
RESUMEN
}

Objetivo: Identificar cómo los adultos mayores que participan en el Proyecto Universidad de Madurez de Amapá (UMAP) experimentan el fenómeno del Síndrome del Nido Vacío. Material y Método: Estudio con enfoque cualitativo, exploratorio y descriptivo, que a través de la narrativa buscó comprender el relato de los participantes. La recopilación de datos se llevó a cabo con siete personas mayores, de edades comprendidas entre 63 y 83 años, a través de entrevistas semiestructuradas, contemplando una pregunta central: cuénteme ¿cómo fue su experiencia cuando sus hijos crecieron y se fueron de casa? Se adoptó el análisis de contenido de Bardin, subsidiado por el software ATLAS.ti versión 8.0. Para garantizar el rigor metodológico se cumplieron los principios de credibilidad, confiabilidad, confirmabilidad y transferibilidad. Resultados: Las tres categorías originadas resultaron de un proceso analítico y explicativo de la experiencia vivida por los ancianos y comprendió tres momentos: la vida invadida por la soledad, el beneficio de la libertad y la toma de nuevos vuelos. Conclusión: La experiencia del nido vacío experimentada por las personas mayores pasó por diferentes fases, sin embargo, la experiencia en UMAP permitió superar la soledad y transformar esta experiencia en una oportunidad de fortalecimiento y crecimiento.

Palabras clave: Persona Mayor; Relaciones Familiares; Síndrome del Nido Vacío; Universidades; Acogimiento.

Data de recepção: 05/08/2020

Data de aceitação: 04/05/2021

\section{INTRODUÇÁO}

A expansão da população idosa constitui ocorrência global e, no Brasil, transcorre de forma bastante acelerada. Projeções do Instituto Brasileiro de Geografia e Estatística (IBGE) indicam que em 2031 a população idosa (43,2 milhóes) irá ultrapassar o quantitativo de crianças e adolescentes de 0 a 14 anos (42,3 milhóes), até o ano de 2042, a população brasileira atingirá 232,5 milhôes de habitantes, onde 57 milhóes será de idosos (24,5\%) e previamente ao ano de 2050, os idosos já representam um contingente superior a parcela da populaçáo com idade entre 40 e 59 anos $^{(1)}$. 
Nesse grupo populacional, a vida de forma autônoma e independente, apesar das limitaçóes biológicas desencadeadas pela progressão da idade, constitui grande desafio, demandando investimento em educação permanente em uma gama de profissionais, com vistas a garantia de um estilo de vida mais favorável à pessoa idosa ${ }^{(2)}$.

Por este ângulo, a Universidade da Maturidade do Amapá (UMAP), instituída como atividade extensionista da Universidade Federal do Amapá (UNIFAP), disponibiliza curso de natureza acadêmica, considerado livre, às pessoas com idade igual ou acima de 60 anos, com vistas à promoção, atualização e qualificação de saberes, de forma a permanecerem agentes atuantes na família e na sociedade.

O aumento da expectativa e a qualidade de vida das pessoas idosas estão aliados não só à evolução da tecnologia e da medicina, mas, também, às suas vivências em grupos, sendo a Universidade Aberta para Maturidade, uma das proposiçóes que vai ao encontro da melhoria da qualidade de vida para os idosos $^{(3)}$.

Ao conceber o envelhecimento como um processo de decréscimo orgânico e funcional não decorrente de doença e que inevitavelmente ocorre com o passar do tempo, buscamos o fenômeno da Síndrome do Ninho Vazio. Estudo registra que o termo "ninho vazio" se refere aos anos que um casal passa juntos entre a saída do último filho de casa e a morte de um dos cônjuges, abrangendo casais com ninhos vazios ou idosos com ninhos vazios ${ }^{(4)}$. Outros autores ressaltam que esse termo ganhou destaque particular durante a década de 1970, estando frequentemente associado à tristeza dos pais após a saída de todos os filhos de casa ${ }^{(5)}$.

Sob uma perspectiva histórica, o ninho vazio constitui fenômeno único e relativamente novo, integrando uma fase importante no desenvolvimento do curso de vida da família. A fase de ninho vazio no ciclo de vida da família era relativamente rara ou de curta duração. Nos tempos contemporâneos, a transiçáo para o ninho vazio é menos linear ou permanente, pois muitos filhos adultos jovens retornam para casa. Estudos interculturais e outros também destacam a diversidade em experiências de ninho vazio por fatores psicológicos e relacionais, origem étnica, classe social, gênero e localidade regional $^{(6)}$.

Ninhos vazios crescerão em número com o envelhecimento da população mundial, estando frequentes associadas à famílias menores, com menor espaçamento entre os filhos e maior longevidade ${ }^{(7)}$.

Diante do exposto, o presente estudo tem como objetivo analisar como idosos integrantes da UMAP vivenciam este período da vida caracterizado como Ninho Vazio.

\section{MATERIAL E MÉTODO}

Trata-se de estudo exploratório, descritivo, qualitativo, tipo investigação narrativa, com o intuito de interpretar significados dos relatos individuais que emergiram durante as entrevistas dos participantes. O estudo vislumbra compreender por meio das narrativas o cotidiano dos participantes diante do fenômeno ${ }^{(8,9)}$.

O estudo foi realizado no período de agosto a novembro de 2019, tendo como participantes idosos de ambos os sexos matriculados na UMAP. Adotou-se como critérios de inclusão: estar matriculado no segundo semestre letivo de 2019, ter idade igual ou superior a 60 anos e não residir com os filhos. Como critério de exclusão: estar de licença médica. Para definição do número de participantes atendeu-se ao critério de saturação dos dados, diante de informaçóes recorrentes durante as entrevistas ${ }^{(10)}$.

A aproximação do pesquisador com os idosos ocorreu durante o acolhimento, onde realiza-se atendimento de enfermagem em sala reservada, proporcionando-se ao idoso cuidados de saúde como: aferição de pressão arterial, teste de glicemia capilar, distribuição de preservativos e orientaçóes básicas à saúde. Dentre os 40 idosos matriculados, 23 buscaram o atendimento de enfermagem, sendo possível identificar que 16 não atendiam aos critérios de inclusão. Deste modo, sete idosos foram convidados a participar do estudo, sendo que nas narrativas de cinco idosos, contemplouse o fenômeno estudado, não havendo nenhuma desistência entre estes.

Para a coleta de dados obteve-se informaçóes sociodemográficas de interesse para o estudo: idade, gênero, número de filhos, naturalidade, raça/cor, estado civil, religião e grau de escolaridade associados a uma entrevista semiestruturada que contemplou uma questão central: Conte-me como 
foi a sua experiência mediante a saída dos filhos de casa.

As entrevistas foram realizadas pelo pesquisador principal em agosto de 2019, em dia e horário de acordo com a conveniência dos idosos, foram realizadas face a face em ambiente reservado na universidade, possibilitando privacidade, conforto e acolhimento. Foram gravadas com aparelho eletrônico para posterior transcrição e tiveram duração entre 15 a 65 minutos.

Os áudios das entrevistas foram transcritos na íntegra utilizando o programa computacional Word e posterior análise de conteúdo, subsidiada pelo software Qualitative Data Analysis (ATLAS. $\left.t i^{\oplus}\right)$ versão 8.0 , desenvolvido especificamente para o armazenamento, manipulação e análise de dados qualitativos, facilitando o gerenciamento dos dados codificados a serem analisados. O ATLAS. $\mathrm{ti}^{\ominus}$ é composto por vários elementos, dentre eles: unidade hermenêutica (arquivo próprio para armazenar as informaçóes no ambiente do software), documentos primários, códigos, citas, famílias, notas de análises e vista de redes (esquemas gráficos). Assim, na organização dos dados deste estudo foi construída uma Unidade Hermenêutica que é o projeto ampliado, constituído de sete documentos primários (entrevistas) e 75 códigos (unidades de registro).

As narrativas foram examinadas por meio de leitura analítica e os códigos foram categorizados de acordo com a semelhança temática e textual como proposto por Bardim ${ }^{(11)}$.

Nesse seguimento, o Atlas.ti possibilitou a organizaçáo dos dados para proceder às etapas de pré análise, codificação, categorização e criação de dimensôes ${ }^{(12)}$. Mediante uma codificação temática, com uma matriz de análise realizada com base no referencial teórico, objetivos e problema de pesquisa, originou-se três dimensóes ou categorias. Fragmentos de texto foram extraídos como unidades de significado. Estes foram codificadas de acordo com sua relevância, que foram analisadas em três categorias temáticas relacionadas através de redes $^{(13)}$.

Para garantir o rigor metodológico utilizouse os princípios de credibilidade, confiabilidade, confirmabilidade e transferibilidade ${ }^{(14)}$. Nesta direção, para a confiabilidade do estudo houve a inserçáo do pesquisador no contexto dos participantes (UMAP); as transcriçóes das entrevistas foram devolvidas aos participantes do estudo para que fossem validadas, propiciando credibilidade; a codificação dos dados foi realizada por dois pesquisadores com auditoria de um terceiro, para a confirmabilidade dos dados adotou-se uma posição reflexiva entre os pesquisadores e com vistas à transferibilidade dos dados, buscouse o conhecimento da forma em que os idosos vivenciaram e se adaptaram ao fenômeno do ninho vazio, com o intuito de expor informaçóes que possibilitem a transferência dos resultados para outros contextos.

Para preservar o anonimato dos participantes do estudo, cada idoso entrevistado recebeu um codinome representado por espécies de pássaros acompanhadas pela idade, simbolizando o momento em que seus ninhos se esvaziaram.

Não houve conflito de interesses no estudo, o qual seguiu as diretrizes da Resolução 466/12 do Conselho Nacional de Saúde, sendo previamente aprovado pelo Comitê de Ética em Pesquisa da Universidade Federal do Amapá (Parecer n. 3.535.850). A decisão de participação foi oficializado por meio da assinatura do Termo de Consentimento Livre e Esclarecido, não ocorrendo recusa dos participantes durante o estudo.

\section{RESULTADOS}

Dados sociodemográficos: Identificaram-se idosos de ambos os sexos, com idades entre 63 a 83 anos, com vínculo religioso, formação escolar predominantemente a nível médio, sendo estes solteiros, viúvos e divorciados. A quantidade de filhos por idoso entrevistados variou entre dois a dez. Este aspecto se faz relevante por evidenciar que um grande número não impede que o ninho se torne vazio mesmo para pessoas que tiveram muitos filhos no decorrer de suas vidas.

Experiência vivenciada: As categorias elaboradas resultaram de um processo analítico e explicativo da experiência vivenciada pelos idosos e compreenderam três momentos: A vida sendo invadida pela solidão, Beneficiando-se da liberdade e Alçando novos voos:

Primeiro momento: A vida sendo invadida pela solidáo. A família constitui um centro de prestação de cuidados. Em um núcleo familiar, 
embora o cuidado se irradie entre os membros da família, a figura da mãe ocupa posição de destaque. Entretanto, quando esse papel já não pode ser exercido de forma plena por esta protagonista do cuidado, pode desencadear sentimento de tristeza, sofrimento e solidão, evidenciados nesta categoria: Foi muito ruim, porque eu fiquei sozinha e não tenho com quem conversar, vivo sozinha noite e dia, tem certas horas que a gente se sente mal, principalmente à noite, não é fácil. Às vezes você fica doente e não tem quem te dê um copo d'água (Andorinha, 76 anos).

Foi só tristeza, porque a gente está acostumada a ver todos os filhos dentro de casa, dai eles vão embora e a gente fica sozinha (Curió, 63 anos).

Quando indagados a revelar sobre as significativas perdas ao longo da vida, nitidamente não se reportaram às perdas do tipo biológicas, ao contrário, as perdas sociais e emocionais produzem mais impactos em suas vidas, principalmente em relação aos filhos, na qual dedicou grande parte de suas vidas:

Foi muito doloroso, pois a gente deseja ter nossos filhos sempre por perto. $\grave{A}$ noite eu me sinto num deserto, num vazio... não tenho ninguém para compartilhar algo [...] você perde a vontade de viver, parece que você só tem vida se for com os filhos, sem os filhos você não é ninguém. Às vezes passo o dia com fome, pois fazer comida só para mim é muito ruim, antes eu era acostumada a cozinhar para muita gente (Sabiá, 65 anos).

Quando não se reconhece os idosos com suas vulnerabilidades e valores próprios, o envelhecimento, muitas vezes, é hostilizado pela sociedade e pelos próprios filhos. Esse padrão de conduta pode agravar sentimentos negativos e de solidão:

[...] parece que a pessoa idosa náo existe para a sociedade, parece que esquecem que vão envelhecer também. Às vezes você entra no ônibus $e$ as pessoas nem levantam para você. Parece que a pessoa idosa não existe! (Andorinha, 76 anos).

Tais ocorrências atestam relacionamentos interpessoais que foram alterados de alguma forma no decorrer de suas vidas, tornando a saída dos filhos do lar um agravante para que o idoso esteja ainda mais suscetível aos sintomas da Síndrome do Ninho Vazio.
Segundo momento: Beneficiando-se da liberdade. $O$ sentimento de liberdade se atribui à quitação de responsabilidades que se deu ao longo da vida destes que agora vivenciam a senectude. Inicialmente, o impacto da saída dos filhos de casa desperta a solidão, entretanto outras experiências surgem no decorrer desse processo, onde o indivíduo idoso reaprende a tomar o controle de sua vida:

[...] antes eu tinha responsabilidades com mulher, filhos, colocar comida dentro de casa. Agora a responsabilidade é só comigo. A partir de quando eu fiquei só, percebi que fiquei dez anos parado, sem fazer nada. Então eu tive a oportunidade de fazer várias coisas que não pude fazer mais cedo (Beija-flor, 83 anos).

[...] anteriormente eu tinha muitas responsabilidades com os meus filhos. Agora não, eu tenho uma vida mais tranquila (Águia, 67 anos).

Neste sentido, evidencia-se certa liberdade para retomar projetos interrompidos ou iniciar novos, por não se encontrar presos a obrigaçóes familiares.

Terceiro momento: Alçando novos voos. Para os idosos, as diversificaçóes das atividades desenvolvidas na UMAP possuem dupla função: possibilita o vínculo e constitui uma atividade terapêutica. A vivência com outros idosos proporciona diálogos, interaçóes e reciprocidade, servindo como apoio para estes que vivenciam a solidão em suas casas, como estão evidenciados nos relatos a seguir:

[...] levei como experiência e aprendi a conviver com a situação, agora me sinto melhor, principalmente depois de descobrir a UMAP. Eu sempre fazia várias coisas, mas eu continuava me sentindo sozinha. Aqui não, eu me sinto acolhida (Sabiá, 65 anos).

[...] a UMAP acaba sendo o meu refúgio, aqui a gente se sente uma familia. Nos finais de semana eu rezo para chegar logo segunda-feira e voltar para cá novamente (Curió, 63 anos).

Em face de um contexto que se reveste de características peculiares, a inserção do idoso em grupos de vivência emerge como uma postura resiliente, caracterizada por um processo de enfrentamento positivo, em que o idoso não sucumbe aos fatores de risco biológico, socioeconômico e 
psicossocial, mas adquire condições regeneradoras que o auxiliam a enfrentar os declínios do processo de envelhecimento.

\section{DISCUSSÁO}

O modo como se vivencia a solidão e como se responde a ela, afeta as atividades diárias e a qualidade de vida do idoso, os quais são particularmente mais vulneráveis a experimentar a solidão e o isolamento social.

Do ponto de vista social, as famílias têm diferentes maneiras de encarar o processo de envelhecimento, cabendo uma melhor compreensão do idoso, sobre seu papel junto à parentela e sua função social enquanto sujeito inserido em um contexto ${ }^{(15)}$.

A literatura destaca que aproximadamente 50\% dos indivíduos com mais de 60 anos estão em risco de isolamento social e um terço experimentará algum grau de solidão na vida ${ }^{(16)}$.

Agrega-se ainda às experiências e sentimentos ocasionados pelo vazio do ninho, a diferença significativa na qualidade do envelhecer de idosos que moram sozinhos, comparado a outros que moram com familiares. Estudo ressalta que idosos que residem sozinhos em seus lares, são mais afetados nos requisitos relacionados à saúde, condiçóes físicas e estado emocional ${ }^{(17)}$.

A solidão constitui estado afetivo vivenciado quando o idoso se percebe socialmente isolado ou possui pouca interação social. Outros estudos registraram que a solidão não está apenas relacionada a resultados adversos para a saúde mental, mas também a uma variedade de condições médicas, como doenças cardiovasculares, diabetes, demência e mortalidade por todas as causas ${ }^{(18)}$.

Dado o crescente ônus da solidão e seu impacto na saúde e no bem-estar, advém-se o interesse público e de políticas em todo o mundo para o contexto do envelhecimento ${ }^{(19)}$. Em países europeus estudo registrou que a solidão nos idosos, relacionase mais com as perdas sucessivas que ocorrem ao longo da vida, do que com a saída dos filhos de casa. Em Portugal, algumas políticas públicas aproximam os jovens da população idosa. Outros países da Europa adotam políticas que investem na inserção dos idosos na sociedade, favorecendo a integraçáo e uma vida ativa, mesmo não sendo laboralmente ativos ${ }^{(20)}$.
No Sul do Brasil, um estudo sobre as influências das relaçóes sociais na sobrevivência dos idosos, mostrou que as interaçóes sociais fortalecidas são marcadores de sobrevivência, portanto, a solidão é um preditor de mortalidade para esse grupo ${ }^{(21)}$.

Por esta razão, as repercussóes advindas dos vínculos sociais são essenciais para o bem estar emocional e afetivo dos idosos, por refletirem positivamente em suas condiçóes de saúde, reduzindo as morbidades ${ }^{(22)}$.

Por outro lado, cabe considerar também que os idosos que superam essa fase de solidão, podem apropriar-se de um estilo de vida mais ativo e independente resultante do redescobrimento de possibilidades de viver sua própria vida com a máxima qualidade possível, que envolve um processo de adaptação às mudanças que ocorrem ao longo da vida relacionadas à manutenção de uma boa senilidade.

Para tanto se faz necessário o envolvimento ativo com a vida, por meio do estabelecimento de relaçóes sociais e atividades produtivas, evidenciando-se assim um conceito ampliado de envelhecimento bem-sucedido ${ }^{(23)}$.

Nesta perspectiva reconhece-se o trabalho das equipes da Atenção Básica/Saúde da Família, as açóes coletivas na comunidade, as atividades de grupo e a participaçáo das redes sociais dos usuários como alguns dos recursos indispensáveis para atuação nas dimensốes cultural e social da pessoa idosa $^{(24)}$.

Estudo Europeu registrou que morar sozinho se configura como oportunidade para o autoconhecimento e estreitamento de laços sociais exteriores ao ambiente doméstico. A partir dessa relação, estudos apontam que projetos educacionais para idosos estimulam a autonomia, melhoram a autoestima, promovem uma melhor qualidade de vida e proporcionam a inserção social das pessoas idosas, além de favorecerem a construção de espaços de convivência e reflexão diante das necessidades de seus integrantes ${ }^{(25,26)}$.

Em face de um grupo etário que se reveste de características peculiares, a adoção de estratégias com vistas à promoçáo do envelhecimento saudável, a Organização Mundial de Saúde concebe a necessidade de ampliação dos serviços de saúde, para garantia de acesso aos serviços centrados nas demandas da populaçáo idosa, maximizando assim a capacidade funcional do idoso ${ }^{(27)}$. 
Assim é nesse contexto que surge a necessidade de evidências sobre a eficácia das intervençóes de promoção da saúde visando minimizar o isolamento social e a solidão entre pessoas mais velhas. Nesse âmbito, um estudo de revisão sistemática apontou que uma intervenção eficaz para combater o isolamento social e a solidão entre as pessoas mais velhas tendiam a ser atividades de grupo a longo prazo, indicando atividades educacionais e sociais como intervençóes específicas que podem aliviar o isolamento social e a solidão entre os idosos ${ }^{(28)}$.

Da mesma forma, outro estudo relatou que características comuns de intervençóes eficazes foram aquelas desenvolvidas no contexto de uma base teórica e aquelas que oferecem atividade social e/ou apoio em formato de grupo. Intervençóes em que os idosos eram participantes ativos também pareciam ser mais eficazes ${ }^{(29)}$.

Tais pressupostos corroboram os achados da literatura que defendem o envelhecimento como um processo adaptativo, que depende da interação de fatores genéticos, biológicos e socioculturais ${ }^{(30)}$.

Este estudo teve como limitação a pesquisa em um único contexto, observando-se a necessidade de ampliar investigaçóes com outros desenhos de pesquisa, envolvendo variáveis socioeconômicas e condiçóes de saúde do idoso. Novos estudos em que sejam analisadas as estratégias utilizadas para socializar o idoso nessa fase da vida, na qual experienciam a Síndrome do Ninho Vazio poderão ampliar as discussóes referentes a este importante tema.

\section{REFERÊNCIAS}

1. Instituto Brasileiro de Geografia e Estatística (IBGE). Número de idosos no Brasil deve dobrar até 2042 [Internet]. 2018 [citado 2019 jun 19]. Disponível em: https://noticias.r7.com/brasil/ numero-de-idosos-no-brasil-deve-dobrar-ate2042-diz-ibge-25072018

2. Souza AOT, Paula ABR, Oliveira FBM. Construção e avaliação de um website sobre saúde do idoso. ReOnFacema [Internet]. 2015 [citado 2021 abr 23]; 1(1): 9-16. Disponível em: http:// www.facema.edu.br/ojs/index.php/ReOnFacema/ article/view/32

3. Peixoto N, Lima LCV, Bittar CML. Percepçóes

\section{CONCLUSÁO}

Em resposta ao objetivo do estudo, foi possível identificar que a experiência do ninho vazio vivenciada pelos idosos perpassou por diferentes fases, em que inicialmente os idosos sofreram intensamente com a solidão, porém, superada esta fase, adaptaram-se a esta experiência e reaprenderam a viver, beneficiando-se da atual liberdade. Em fase posterior, foi possível dirigir o olhar para o processo de resiliência conquistado pelos idosos, evidenciando que a liberdade os inseriu em grupos de vivência com outros idosos, tornando a UMAP um ambiente acolhedor, propiciando o estabelecimento de novos relacionamentos interpessoais.

O aumento da expectativa de vida da população brasileira é um fenômeno que necessita de políticas públicas que favoreçam a qualidade do envelhecer. Pouco se fala na literatura sobre as necessidades psicossociais dos idosos, sendo muitas vezes o foco principal de determinadas patologias as quais estejam acometidos e este estudo enfatiza que projetos como a UMAP contribuem para a qualidade do envelhecer dos idosos no Amapá, podendo ser exemplo para os estados brasileiros. Alcançar a senectude é uma grande vitória e vivêla com saúde mental e física é imprescindível, nesta direção, a UMAP constituiu uma estratégia que viabilizou superar a solidão e transformar esta experiência em oportunidade de fortalecimento e crescimento.

sobre qualidade de vida entre idosos que participam de uma Universidade Aberta para Maturidade. Acta Scientiarum. Human and Social Sciences [Internet]. 2017 [citado 2021 abr 27]; 39(2): 209-16. Disponível em: https://doi.org/10.4025/ actascihumansoc.v39i2.33089

4. Barber CE. Transition to the empty nest. In Ageing and the Family; Bahe SJ, Peterson ET, Eds.; Lexington Books: Lexington, MA, USA, 1989; [citado 2021 abr 23]; 15-32 p. Disponível em: https://www.researchgate.net/publication/232522 690

5. Rosen E, Ackerman L, Zosky D. The sibling empty nest syndrome: The experience of sadness as siblings leave the family home. J Hum Behav Soc Environ 
[Internet]. 2002 [citado 2021 abr 23]; 6(1): 65-80. Disponível em: https://www.tandfonline.com/doi/ abs/10.1300/J137v06n01_05

6. Mitchell BA. Empty Nest. In: The Wiley Blackwell Encyclopedia of Family Studies. Chapter [Internet] 2016 [citado 2021 apr 25]. Disponível em: https:// www.researchgate.net/publication/314924162_ Empty_Nest

7. Costa DL. Displacing the family: Union army pensions and elderly living arrangements. J Political Econ [Internet]. 1997 [citado 2021 abr 23]; 105(6): 1269-92. Disponível em: https://doi. org/10.1086/516392

8. Pereira EBF, Eugenio BG. Narrativas de formação: potencialidades e possibilidades para a pesquisa em educação. e-Mosaicos [Internet]. 2019 [citado 2021 abr 25]; 8(18): 31-46. Disponível em: https:// doi.org/10.12957/e-mosaicos.2019.41983

9. Damasceno NFP, Malvezzi E, Sales CM, Sales A. Narratives as alternative in health research. Interface [Internet]. 2018 [citado 2021 apr 23]; 22(64): 133-40. Disponível em: 10.1590/180757622016.0815

10. Minayo MCS. Amostragem e Saturação em Pesquisa Qualitativa: consensos e controvérsias. Rev Qualitat [Internet]. 2017 [citado 2021 abr 23]; 5(7): 1-12. Disponível em: https://editora. sepq.org.br/index.php/rpq/article/view/82/59

11. Bardin L. Análise de Conteúdo. São Paulo: Ediçôes 70; 2011. 229 p.

12. Angrosino M. Etnografía y Observación Participante en Investigación Cualitativa. $1^{\mathrm{a}}$ ed. Madrid: Morata; 2012. $144 \mathrm{p}$.

13. Gibbs GR. Different Approaches to Coding. Sociol Methodol [Internet]. 2012 [citado 2020 jul 22]; 42(1): 82-4. Disponível em: https://journals. sagepub.com/doi/10.1177/0081175012460853

14. Lincoln YS; Guba EG. Naturalistic inquiry. 1ª ed. New York: Sage; 1991. 416 p.

15. Dos Santos SE, Fernandes MA, Moura MEB, Almeida CAPL. Elementos da formação do enfermeiro na prevenção da violência contra a pessoa idosa. Cienc enferm [Internet]. 2019 [citado 2021 abr 25]; 25: 7. Disponível em: http://revistasacademicas.udec.cl/index.php/ cienciayenfermeria/article/view/1317

16. Landeiro F, Barrows P, Nuttall Musson E, Gray A, Leal J. Reducing social isolation and loneliness in older people: a systematic review protocol. BMJ Open [Internet]. 2017 [citado 2021 apr 25]; 7: e013778. Disponível em: https://doi 10.1136/ bmjopen-2016-013778

17. Negrini ELD, Do Nascimento CF, Da Silva A, Antunes JLF. Elderly persons who live alone in Brazil and their lifestyle. Rev bras geriatr gerontol
[Internet]. 2018 [citado 2021 Apr 26]; 21(5): 523 31. Disponível em: https://doi.org/10.1590/198122562018021.180101

18. Christiansen J, Larsen FB, Lasgaard M. Do stress, health behavior, and sleep mediate the association between loneliness and adverse health conditions among older people? Soc Sci Med [Internet]. 2016 [citado 2021 apr 26]; 152: 80-6. Disponível em: https://doi.org/10.1016/j.socscimed.2016.01.020_

19. Teixeira SM. Envelhecimento, família e políticas públicas: em cena a organização social do cuidado. Ser Soc Soc [Internet]. 2020 [citado $2021 \mathrm{abr}$ 26]; 137: 135-54. Disponível em: http://dx.doi. org/10.1590/0101-6628.205

20. Rodrigues RM. Solidão, um fator de risco. Rev Port Med Geral Fam [Internet]. 2018 [citado 2021 abr 26]; 34(5): 334-38. Disponível em: 10.32385/ rpmgf.v34i5.12073

21. Soares MU, Facchini LA, Nedel FB, Wachs LS, Kessler M, Thumé E. Social relationships and survival in the older adult cohort. Rev Latino-Am Enfermagem [Internet]. 2021 [citado 2021 apr 26]; 29: e3395. Disponível em: 10.1590/15188345.3844.3395

22. Valtorta NK, Moore DC, Barron L, Stow D, Hanratty B. Older Adults' Social Relationships and Health Care Utilization: A Systematic Review. Am J Public Health [Internet]. 2018 [citado 2021 Apr 28]; 108(4): e1-e10. Disponível em: 10.2105/ AJPH.2017.304256

23. Vitorino SS, Miranda MLJ, Witter C. Educação e envelhecimento bem sucedido: reflexôes sobre saúde e autocuidado. Rev Kairós [Internet]. 2012 [citado 2021 abr 25]; 15(3): 29-42. Disponível em: https://revistas.pucsp.br/index.php/kairos/article/ view/7531/10183

24. Brasil. Ministério da Saúde (MS). Secretaria de Atenção à Saúde. Departamento de Atenção Básica: Envelhecimento e saúde da pessoa idosa. Brasília; Ministério da Saúde [Internet]. 2006 [citado 2021 abr 26]. 192p. Disponível em: https://bvsms.saude. gov.br/bvs/publicacoes/evelhecimento_saude_ pessoa_idosa.pdf

25. Wichmann FMA, Couto AN, Areosa SVC, Montanes MCM. Grupos de convivência como suporte ao idoso na melhoria da saúde. Rev Bras Geriatr Gerontol [Internet]. 2013 [citado $2021 \mathrm{abr}$ 25]; 16(4): 821-25. Disponível em: https://doi. org/10.1590/S1809-98232013000400016

26 Sixsmith J, Sixsmith A, Fänge AM, Naumann D, Kucsera C, Tomsone S, et al. Healthy ageing and home: the perspectives of very old people in five European countries. Soc Sci Med [Internet]. 2014 [citado 2021 apr 26]; 106: 1-9. Disponível em: https://doi.org/10.1016/j.socscimed.2014.01.006 
27. Who. World Health Organization. World report on ageing and health. (NLM classification: WT 104). Geneva: WHO Library [Internet] 2015 [citado 2020 apr 26]. Disponível em: http://apps. who.int/iris/bitstream/handle/10665/186463/ 9789240694811_eng.pdf?sequence $=1$

28. Both JE, Leite MT, Hildebrandt LM, Pilati ACL, Stamm B, Jantsch LB. Grupos de convivência: uma estratégia de inserção do idoso na sociedade. Rev Contexto Saúde [Internet]. 2011 [citado $2021 \mathrm{abr}$ 25]; 10(20): 995-8. Disponível em: https://doi. org/10.21527/2176-7114.2011.20.995-998
29. Dickens AP, Richards SH, Greaves CJ, Campbell $\mathrm{JL}$. Interventions targeting social isolation in older people: a systematic review. BMC Public Health [Internet]. 2011 [citado 2021 abr 25]; 11: 647. Disponível em: http://www.biomedcentral. com/1471-2458/11/647

30. Silva JEG, Eulálio MC, Souto RQ, Santos KL, Melo RLP, Lacerda AR. The capacity for resilience and social support in the urban elderly. Revista Cien Saúde Colet [Internet]. 2019 [citado 2021 abr 25]; 24(1): 7-16. Disponível em: 10.1590/141381232018241.32722016 